\title{
The Influence of the Indenter Tip-Radius on Indentation Testing of Brittle Materials
}

\author{
M. Ciavarella* and D. A. Hills \\ Department of Engineering Science, Oxford University, Parks Road, Oxford OX1 3PJ UK
}

(Received 30 March 1998; accepted 31 July 1998)

\begin{abstract}
Indentation testing of a brittle material using a notionally 'sharp' indenter may reveal several important physical properties, including fracture toughness, surface finish information and the residual stress state. In the case of shallow cone indenters, the contact and fracture mechanics is well defined and closed-form solutions exist in elasticity theory. However, no real indenter is atomically sharp, and the scope of the present article is to quantify how a finite apex radius may modify the stress state induced by a conical indenter. In particular, implications for the load-displacement relation, occurrence of yielding and maximum contact pressure induced are found. A brief discussion of the influence of edge radius on the flat-ended indenter, once used to induce Hertzian type ring cracks, is also included, as this may be treated by a similar procedure. (C) 1998 Elsevier Science Limited. All rights reserved
\end{abstract}

Keywords: indentation testing, fracture, toughness, mechanical properties, surfaces.

\section{Introduction}

Indentation testing of brittle or semi-brittle materials, such as ceramics or glasses, provides the potential to measure important surface characteristics such as fracture toughness, characteristic flaw size distributions, or residual stress states. What is revealed depends partly on what is assumed, but the choice of test geometry is particularly important, and the first decision is to use a 'sharp' or a 'blunt' indenter. In the first category (sharp indenters), are a Vickers pyramid, a Berkovtich pyramid, or a conical indenter. This group has the important

*To whom correspondence should be addressed. Fax: + 44(0)1865-279-802; e-mail:michele.ciavarella@eng.ox.ac.uk property that a limited amount of plasticity is provoked at the vertex of the indenter, which produces a tensile residual stress field in the unloading phase, promoting crack extension. The non-axisymmetric configurations are by far the most commonly used, and the indenter shape is also too remote from the elastic half-space for this idealization to be used in a model. The corresponding analysis of the indentation itself and any subsequent fracture process cannot be more than approximate ${ }^{1}$ or numerical: at best, only an extremely intensive FE numerical analysis can model a 3D elasto-plastic contact. ${ }^{2,3} \mathrm{~A}$ much more detailed analysis is, however, possible for the case of a axisymmetric sharp but shallow, conical indenter. ${ }^{4}$ The subsequent crack problem can also been studied, with the hypothesis that the crack itself does not modify significantly the contact problem: for generally curved 3-D cracks, particularly those originating from small defects in the material, the eigenstrain method is appropriate see Refs 5 and 6, Chapters 6 and 7). Analysis using this approach has been conducted on Hertzian cracking. ${ }^{7-9}$ Generally a surface flaw will 'run around' to form a shallow ring crack before propagating inwards into the material. ${ }^{9}$ If a ring or cone crack forms, further advantage may be taken of the axisymmetry of the crack problem, by employing ring dislocations (see Ref. 6, chapter 5).

In the present article we give the stress state induced by a conical indenter, in the case where the apex of the indenter is in the form of a spherical cap [Fig. 1(a)], which may be thought of as an idealization of the blunting of a true cone. ${ }^{10}$ It is worth remarking that, as the load is increased, the contact problem will start off as a Hertzian configuration, will move through a transition state in which both the tip radius and the conical indenter surface play key rôles, and finally asymptotically tend towards the solution for a true conical indenter. It follows that, at light loads, the stress state induced will be Hertzian, and, unless the material is exceptionally soft, in which case the use of an 


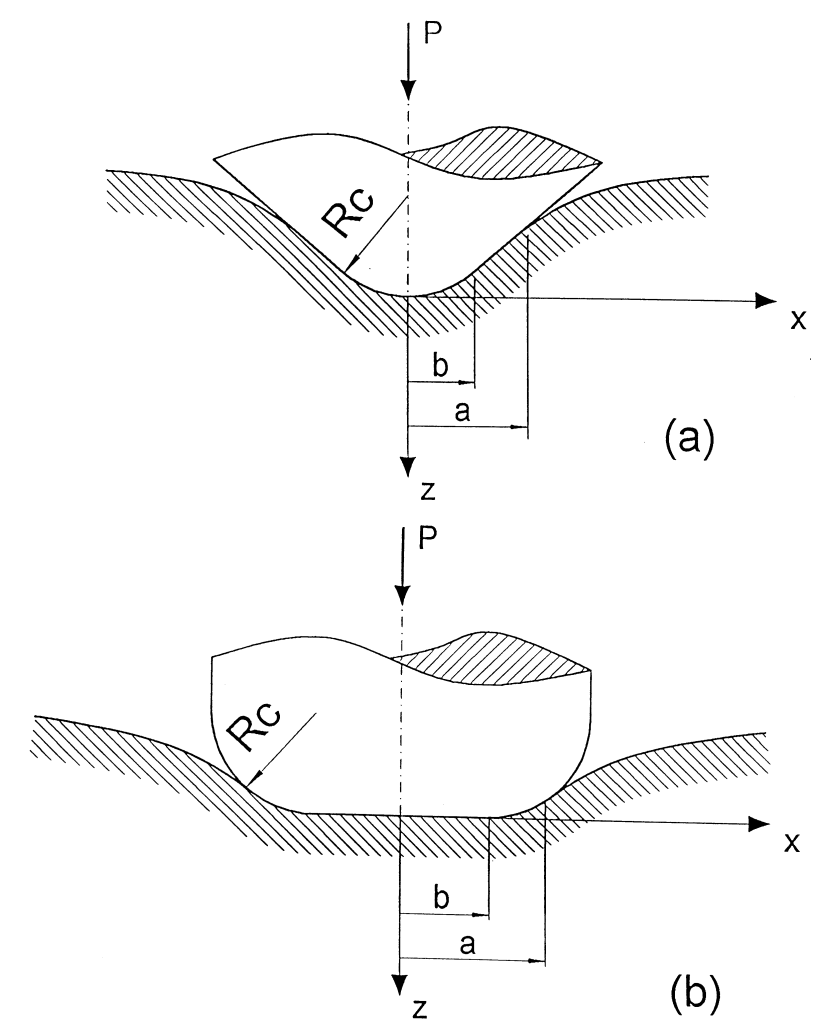

Fig. 1. Geometry of the indentation problem: (a) conical punch; (b) flat punch.

elasticity formulation is inappropriate, the stress state will be entirely elastic, and given by Hüber ${ }^{11}$ (see Ref. 12, Section 7.2). The pressure singularity will, of course, be suppressed, and we would expect that there will be a gradual, smooth transition to the mixed-geometry problem, as the contact disk moves onto the straight part of the indenter profile. It is worth remarking that in indentation tests on brittle materials, there are two key parameters to consider; the first is the maximum tension in the surface, as this is the quantity which is responsible for starting and propelling surface defects, and it is known that the maximum value of $\sigma_{r r}$ occurs at the edge of the contact disk. Further, from a theorem due to Way, ${ }^{13}$ it is known that the surface stress state exterior to an axisymmetric frictionless contact is independent of the actually pressure distribution. Thus, the underlying surface tension does not depend on the exact profile of the indenter. The second effect is the presence of any plasticity, and the way in which it develops. Although the tests to which we wish to apply these results are essentially elastic, it is known and accepted that, for sharp indenters, the presence of localised plasticity, normally at the indenter apex, and occurring during the loading phase, produces residual stresses which are tensile in character, and which are of great importance in propelling cracks as the load is removed.

The external angle of the cone is a particularly important variable: if it is small enough for the plasticity induced at the vertex to be negligible (in the limiting case, a flat punch results), the surface stress state induces Hertzian cracking. The elastic solution usually adopted for a shallow, sharp, conical or flat indenter (that due to Sneddon, ${ }^{14}$ in the case of the shallow sharp cone, and that due to Boussinesq (see, e.g. Ref. 15 for the flat rigid indenter) predict singularities in the stress field (logarithmic, or inverse square root, respectively), and the presence of plastic flow seems inevitable. However, when a blunted cone is considered, it is apparent that plastic flow may not occur and Hertzian cracking may be prevalent.

\section{Formulation}

The problem will be formulated as two contacting half-spaces (Fig. 1), which may, in general, be of different elastic properties but it will be assumed that no interfacial shearing tractions arise, so that the interface must either be lubricated, or the following relation must hold:

$$
\frac{1-2 v_{1}}{\mu_{1}}=\frac{1-2 v_{2}}{\mu_{2}}
$$

where $\mu_{i}$ is the modulus of rigidity and $v_{i}$ Poisson's ratio of body $i$. The use of the half-space formulation is justifiable, providing that the external cone angle is small, and the use of small-strain elasticity theory is acceptable providing that the indentation depth is small compared with the radius of the contact disk.

Well-established techniques for the solution of integral equations for elastic half-spaces can be used. ${ }^{12,16,17}$ The governing integral equation and the main results of the contact problem for this geometry are reported in Ref. 10 which gives the pressure distribution $p(r)$ over the contact area $S$, of radius $a$, and in this paper we will confine ourselves to a summary of the main results relevant to indentation testing.

\subsection{Conical punch}

In the case of the conical punch, [Fig. 1(a)], the geometry is defined by

$$
z(r)=\left\{\begin{array}{cc}
\frac{\theta}{2 b} r^{2}, & \phi \leq r \leq b, \\
\theta r-\frac{\theta}{2} b, & b \leq r \leq a
\end{array}\right.
$$

where $\theta$ is the external angle of the cone. As this must be small $\theta \simeq \tan \theta$. Note, then, that $\theta / b=k=1 / R_{c}$. We assume that the load is sufficiently heavy for the contact disk to envelope the spherical cap, i.e. $a>b$. For this problem it is simplest to start with an evaluation of the contact law, which gives ${ }^{10}$ 


$$
\begin{aligned}
\frac{A P}{4 \theta} & =\frac{b^{2}}{6 \cos ^{3} \varphi_{0}}\left[4+3 \varphi_{0} \cos \varphi_{0}\right. \\
& \left.-3 \sin \varphi_{0}-\sin ^{3} \varphi_{0}\right]
\end{aligned}
$$

where $\cos \varphi_{0}=\frac{b}{a}$, and $A$ as the 'composite compliance' of the bodies, defined by

$$
A=\frac{1-v_{1}}{\mu_{1}}+\frac{1-v_{2}}{\mu_{2}}
$$

The approach, $\alpha_{n}$, is obtained ${ }^{10}$ as

$$
\alpha_{n}=\frac{b \theta}{\cos ^{2} \varphi_{0}}\left(1-\sin \varphi_{0}+\varphi_{0} \cos \varphi_{0}\right)
$$

Finally, the non-dimensionalized pressure distribution is given in quadrature in Ref. 10 but is not reported here. The requirement that $a>b$ corresponds to a minimum load of

$$
\frac{A P}{\theta}=\frac{8}{3} b^{2}
$$

Notice that for $\varphi_{0} \simeq 0$, first order approximations for the load and approach are given by

$$
\frac{A P}{\theta} \cong \frac{8 b^{2}}{3}\left[1-\frac{\varphi_{0}^{3}}{4}\right] ; \alpha_{n}=b \theta
$$

Fig. 2 shows the pressure distribution for $b / a=0,0.2, \ldots, 1$. It may be appreciated that, as $b / a \rightarrow 0$ the maximum pressure (at the contact centre $r=0$ ) becomes increasingly high; indeed, it grows logarithmically, in the form ${ }^{10}$

$$
\frac{p_{\max }}{p_{m}}=\frac{6 \cos \varphi_{0}}{4+3 \varphi_{0} \cos \varphi_{0}-3 \sin \varphi_{0}-\sin ^{3} \varphi_{0} \Psi\left(\varphi_{0}\right)}
$$

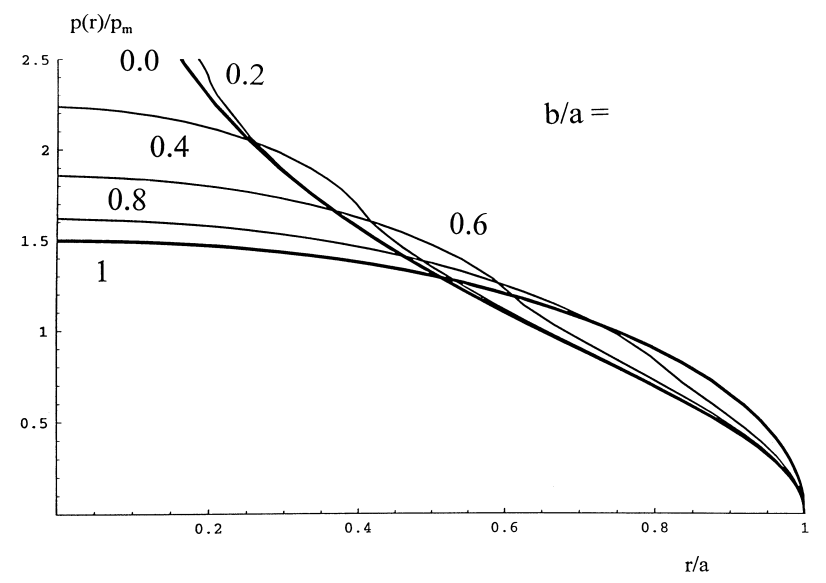

Fig. 2. Pressure distribution $p\left(\frac{4}{a}\right) / p_{m}$ for a conical indenter with rounded apex: $(b / a=0 \cdot 0,0 \cdot 2, \ldots 1)$. Bold lines correspond to the limiting cases: a sharp conical punch when $b / a=0$ and Hertzian when $b / a=1$. where*

$$
\Psi\left(\varphi_{0}\right)=\sec \varphi_{0}+\varphi_{0}-\tan \varphi_{0}+\frac{1}{2 \int_{0}^{\varphi_{0}} \varphi \tan \varphi \mathrm{d} \varphi}
$$

and $\frac{p_{\max }}{p_{m}}$, the ratio of maximum to mean contact pressure, is plotted in Fig. 3. It may be noted that the maximum pressure is just double the Hertzian value $\left(\frac{p_{\max }}{p_{m}}=1.5\right)$ for an indenter such that $b / a$ approx $0 \cdot 2$.

\subsection{Flat punch}

We will deal briefly with the problem of indentation by a flat-ended punch, having a small radius, $R_{c}$, present around the outer edge, [Fig. 1(b)]. This type of indenter was used by Roesler ${ }^{18}$ to induce 'Hertzian' type cone cracking, although the influence of the precise geometry of the indenter was not addressed in the original paper. However, the technique described here is appropriate for analyzing this geometry, providing the contact does not extend into the rounded part far enough for the elastic half-space idealization to be called into question; the results are included here as they also provide an example of a singular pressure distribution being replaced by a smooth, bounded one, because of the removal of a sharp corner.

In this case, the profile of the geometry is described simply by

$$
z(r)=\left\{\begin{array}{ll}
\theta, & 0 \leqslant r \leqslant b \\
\frac{k}{2}(r-b)^{2}, & b \leqslant r \leqslant a
\end{array}\right\}
$$

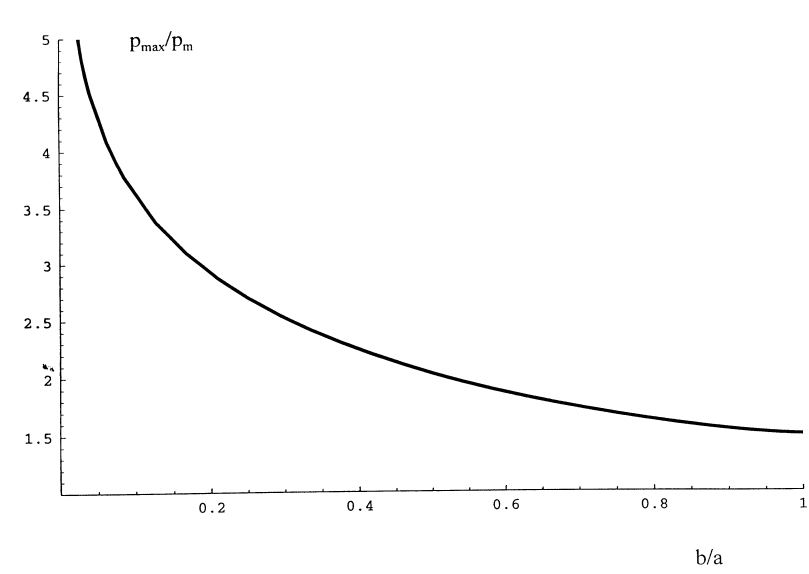

Fig. 3. Maximum pressure $p_{\max } / p_{m}$ for a conical indenter with rounded apex, as a function of $b / a$.

The remaining integral cannot be found in closed form, and can be given in series ${ }^{19}$

$$
\begin{aligned}
& \int x \tan x \mathrm{~d} x=\sum_{k=1}^{\infty}(-1)^{k+12^{2 k}(2 k-1)} \frac{B^{k k(2 k) !}}{2 k} x^{2 k} \\
& \text { where } B_{o}=0 \text { and } B_{k}=\sum_{n=0}^{k}\left(\frac{k}{n}\right) B_{n}
\end{aligned}
$$


where $k$ is the curvature of the rounded part, i.e. $k=\frac{1}{R_{c}}$, see [Fig. 1(b)]. The contact load $P$ is given by ${ }^{10}$

$$
P=\frac{2 k b^{3}}{3 A} \frac{3 \sin \varphi_{0}+\sin ^{3} \varphi_{0}-3 \varphi_{0} \cos \varphi_{0}}{\cos ^{3} \varphi_{0}}
$$

and the auxiliary angle $\varphi_{0}$ is again given by $\cos \varphi_{0}=\frac{b}{a}$. The approach $\alpha_{n}$, is obtained as

$$
\alpha_{n}=k b^{2} \frac{\tan \varphi_{0}-\varphi_{0}}{\cos \varphi_{0}}
$$

Finally, the pressure distribution is given in quadrature in Ref. 10 Here the explicit solution is not reported, but the pressure distribution for several values of the ratio $b / a$ is plotted in Fig. 4 . The maximum pressure is not achieved at a fixed point, so no simplified expression is possible, as was the case of the rounded conical indenter.

Figure 5 shows simultaneously the variation of the non-dimensional load $\frac{A P R_{c}}{a^{3}}$, and non-dimensional remote approach $\frac{R_{c} \alpha_{n}}{a^{2}}$, with $b / a$, for the two types of indenter. The results for a conical indenter are the ascending lines and those for the flat indenter are the descending lines. Note that the Hertzian value corresponds to $b / a=0$ and $b / a=1$, for the flat and conical case, respectively.

\section{Stress Field Induced}

An explicit calculation of the interior stress field is described here using a direct method, which gives rise to a rapidly convergent numerical integration* The technique appeals directly to potential theory (see Ref. 20, for further details). It may be shown

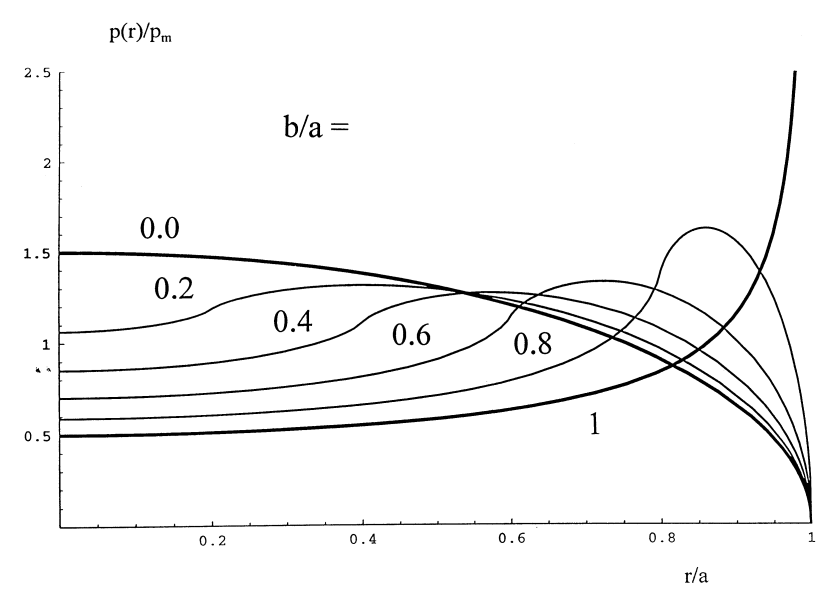

Fig. 4. Pressure distribution $p\left(\frac{r}{a}\right) / p_{m}$ for a flat indenter with rounded corners: $(b / a=0 \cdot 0,0 \cdot 2, \ldots 1)$. Bold lines correspond to the limiting cases: a Hertzian punch when $b / a=0$ and a rigid, sharp, flat punch when $b / a=1$.

*Except for the region of the surface contact disk, where numerical care is required because of the nearly singular or singular integrals to be computed. that the stress and displacement fields can be obtained by defining an harmonic potential, $N$, which is related to the stress field by

$$
\begin{gathered}
\sigma_{x x}-N_{x x}-2 v N_{y y}-z N_{x x z} \\
\sigma_{y y}=-N_{y y}-2 v N_{x x}-z N_{y y z} \\
\sigma_{z z}=-N_{z z}-z N_{z z z} \\
\sigma_{x y}=-(1-2 v) N_{x y}-z N_{x y z} \\
\sigma_{y z}=-z N_{y z z} \\
\sigma_{z x}=-z N_{x z z}
\end{gathered}
$$

where the subscripts indicate derivatives. The potential $N$ itself is defined in Refs. 10 and 12 from the 'stress field' function $g(t)$ which is a real function of $t$, obtainable from either the pressure or the undeformed surface profile as (Ref. 12, eqns (6.11 and 7.23))

$$
g(t)=\frac{2}{\pi} \frac{d}{\mathrm{~d} t} \int_{t}^{a} \frac{r p(r) \mathrm{d} r}{\sqrt{r^{2}-t^{2}}}=\frac{2}{\pi A} \frac{\mathrm{d}^{2}}{\mathrm{~d} t^{2}} \int_{0}^{t} \frac{r z(r) \mathrm{d} r}{\sqrt{t^{2}-r^{2}}}
$$

but here the second option will be used as it is more convenient. Once $g(t)$ is known, it is relatively straightforward to compute the entire stress field. Details of the potentials needed and their derivatives for the determination of the stress field from eqns (13-15) are given in Refs 12 and 21, and also in Ref. 10

For the rounded conical indenter, using (19) and (2), when the contact disk extends onto the conical frustum, we get

$$
\begin{aligned}
g(t) & =\frac{2 \theta}{\pi A b}\left[2 t-2 \sqrt{t^{2}-b^{2}}\right. \\
& \left.+b \arccos \frac{b}{t}\right] ; \quad t>b
\end{aligned}
$$

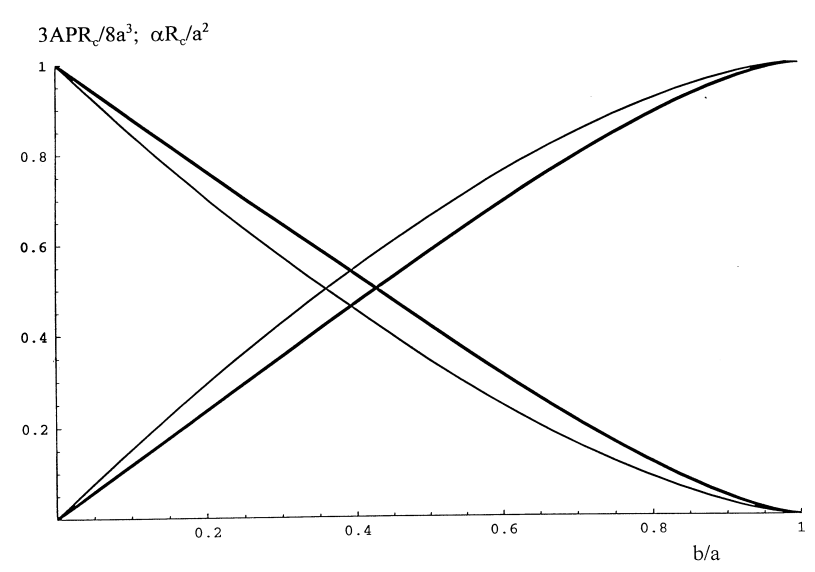

Fig. 5. Non-dimensional load $\frac{A P R_{c}}{a^{3}}$ (bold line) and nondimensional approach $\frac{R_{c} a_{n}}{a^{2}}$, for a conical indenter (ascending lines) or flat indenter (descending lines) as a function of $b / a$. 
whereas if the contact is entirely within the spherical cap, we recover Hertz' solution, which, in terms of the variables currently being used, is

$$
g(t)=\frac{\theta}{\pi A b} t ; t<b
$$

Note, too, that this solution may be recovered by taking the limit as $b \rightarrow a$, whereupon we re-obtain the result in the form $g(t)=\frac{4 \theta}{\pi A}\left(\frac{t}{a}\right)$. Lastly, by taking the opposite limit, i.e. $b \rightarrow 0$, or, in other words, making the tip radius vanishingly small, we obtain the conical punch value $g(t)-\frac{h(t)}{f}=\frac{\theta}{A}$. Turning, now, to the flat indenter with radiused edge, when the contact extends into the rounded region (as it will for any finite load and non-zero radius) we obtain from (19)

$$
g(t) \frac{2 k}{\pi A}\left[2 \sqrt{t^{2}-b^{2}}-b \arccos \frac{b}{t}\right] ; t>b
$$

If the edge radius is reduced to zero the result for a flat-ended punch is recovered, i.e.

$$
(t)=0 ; t<b
$$

For completeness, we note that in the limit as $b \rightarrow 0$, we again re-obtain the Hertzian value $g(t)=\frac{4 k}{\pi A} t$

\section{Implications for Indentation Testing}

We begin by re-iterating a remark made in the introduction; that surface defects are initially propelled by the most positive principal stress lying in the surface, which, for frictionless axi-symmetric indenters lies at the edge of contact $r=a$, and is in the $r$-direction. The theorem due to $\mathrm{Way}^{13}$ dictates that the stress on the surface is the same for all indenter profiles, and is given by

$$
\frac{\sigma_{r r}}{p_{m}}=\frac{1-2 v}{2}\left(\frac{a}{r}\right)^{2}, r>a
$$

so that the maximum value, at $r=a$, lies between 0 , for an incompressible material $(v=1 / 2)$, and $1 / 2$ for a material showing no Poisson effect $(v=0)$. Hence, the tendency to start a small surface crack growing does not depend on the profile, itself. However, it should be recognized that the variation of contact radius, $a$, with applied load differs from profile to profile, so that this does not imply that the maximum tension will be the same for a given applied contact force.

Further, the interior stress field does depend on the profile, and limited results are displayed for the blunt conical indenter in Fig. 6[(a) for Poisson's ratio equal to 0 (b) for Poisson's ratio equal to $0 \cdot 3]$. The difference in the radial component of the stress field (which gives a clear indication of the state of affairs for radial cracking mode) depends strongly on profile only in the region $0 \leq z / a \leq 0 \cdot 4$, and the variation is never particularly great. For the case of a rounded flat indenter the variation is more significant, and is given in Fig. 7 The limiting case of a perfectly square edged indenter predicts an infinitely high concentration of compression at the edges.

\subsection{Yielding}

We will now turn our attention to the question of yielding. Even for a nominally elastic contact, the question of yielding is important for two reasons; first, because we wish to investigate whether plastic flow will precede the fracture process, with the possibilities of re-distribution of both internal stress field and, indeed, contact pressure, and secondly, because tensile residual stresses are important in propelling cracks initiated during the unloading phase.
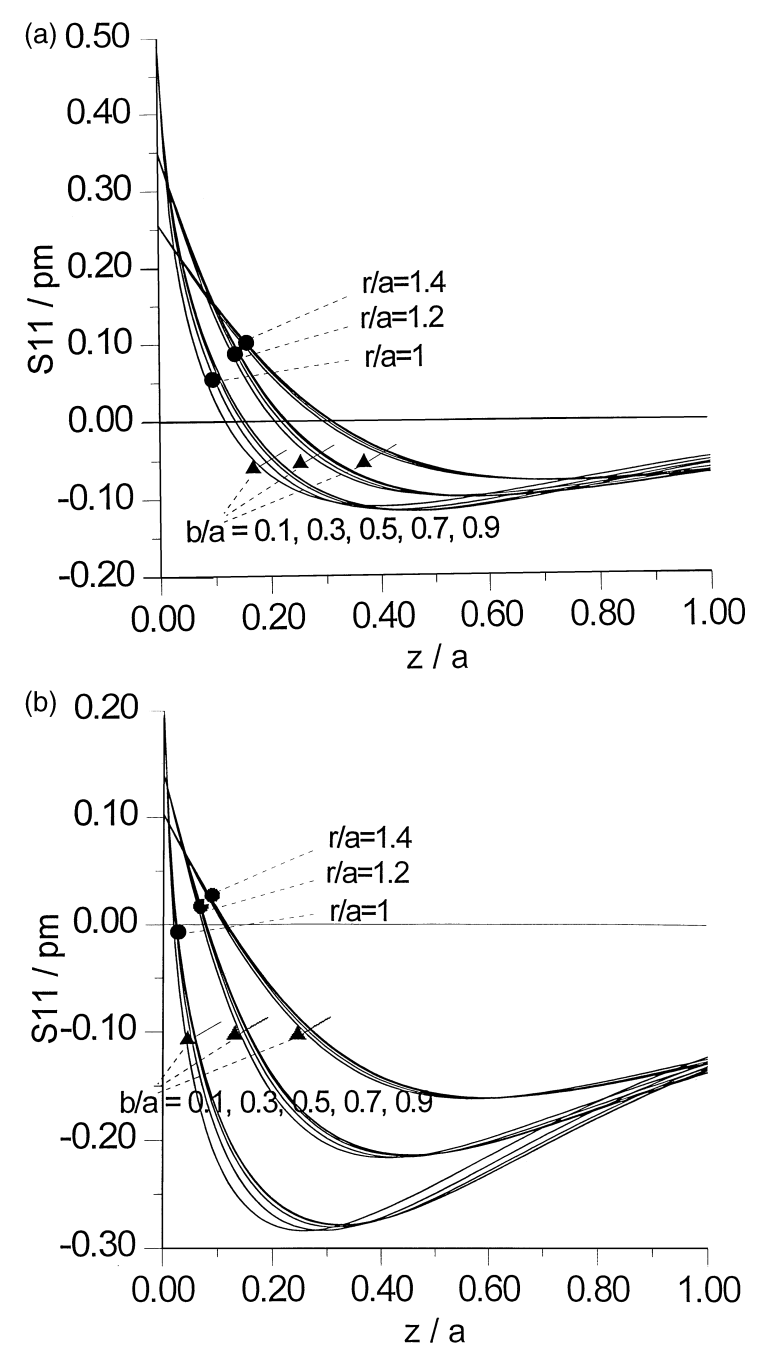

Fig. 6. Radial stress distribution subsurface for conical indenter: (a) $v=0 \cdot 0 ;$ (b) $v=0 \cdot 3$ 


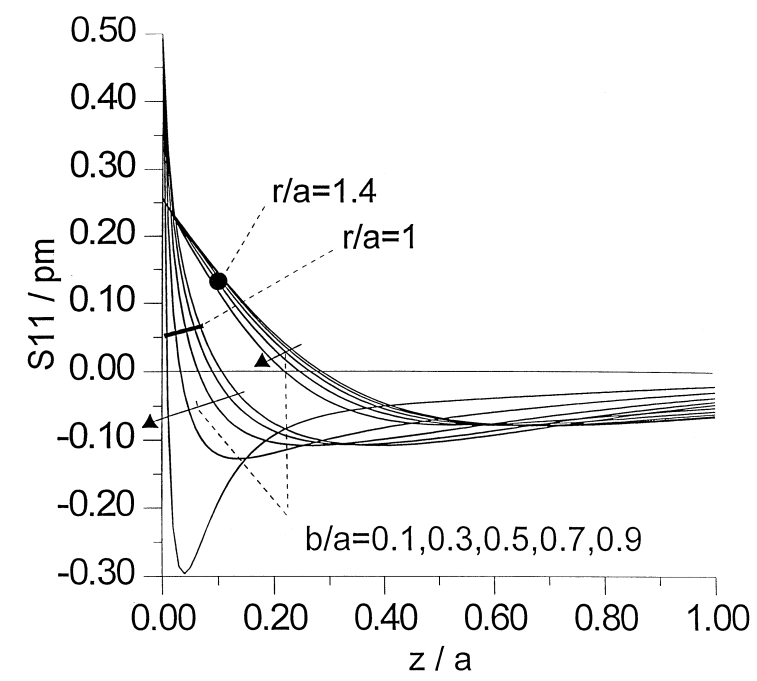

Fig. 7. Radial stress distribution subsurface for flat indenter $(v=0 \cdot 0)$.

Figure 8 includes a plot of the normalized elastic limit $P /\left(a^{2} k\right)$, where $k$ is the yield strength in pure shear, as a function of the ratio $b / a$. For the radiused cone, the strength is always lower compared than the Hertz limiting case, as expected, as the pressure distribution is localized near the centre of the contact. The elastic limit in the sharp cone case is theoretically zero, unless $v=0 \cdot 5 .^{22}$

Turning to the case of flat indenter, we have remarked that the radial stress field induced is very sensitive, subsurface, to the actual value of $b / a$, and for nearly sharp indenters the tensile field has very steep gradients which render it less attractive in many respects. Figure 9 shows that varying $(b / a)$ causes the strength of the contact first to increase with respect to the Hertzian case, then to decrease very slowly. According to von Mises' yield criterion, the strength is in fact higher than the

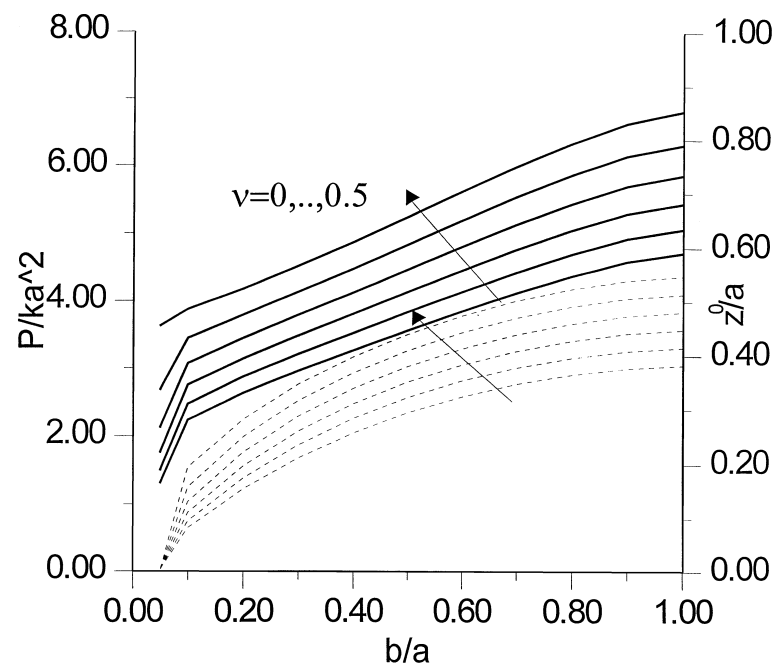

Fig. 8. Elastic limit $\frac{p}{k a^{2}}$, for indentation with a conical indenter, as a function of $b / a$ (solid lines). The upper limit $(b / a=1)$ is Hertzian indenter. $(v=0 \cdot 0,0 \cdot 1, \ldots 0 \cdot 5)$. Also indicated the vertical position $z 0 / a$ of the point of first yielding (broken lines).

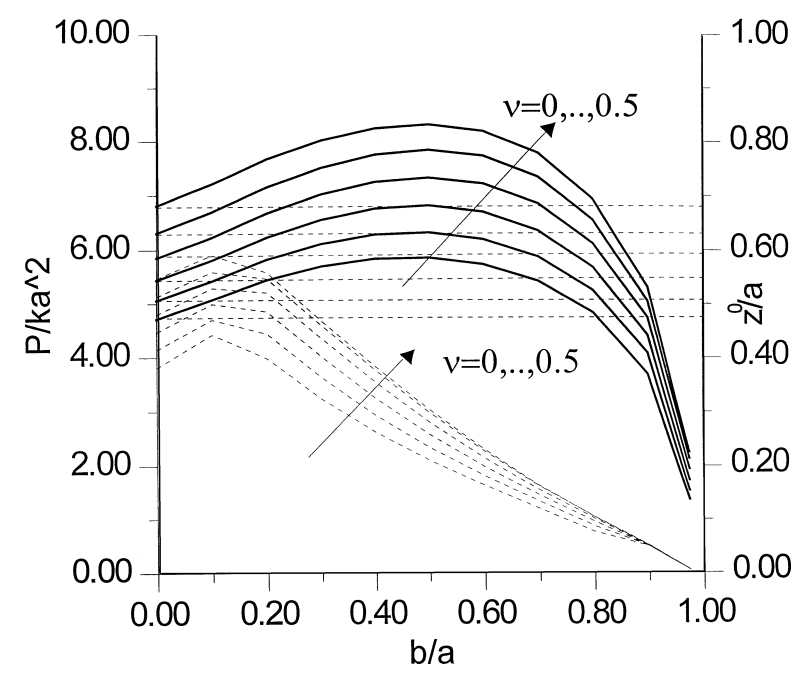

Fig. 9. Elastic limit $\frac{p}{k q^{2}}$, for indentation with a flat indenter, as a function of $b / a$ (solid lines). The upper limits $(b / a=0)$ are relative to Hertzian indenter, and are indicated also with horizontal broken lines. The strength is higher than Hertzian for $b / a<0.8$ (about). Also indicated the vertical position $z 0 / a$ of the point of first yielding (broken lines) $(v=0 \cdot 0,0 \cdot 1, \ldots 0 \cdot 5)$.

Hertzian case over the approximate range $0<b / a<0 \cdot 8$ for all Poisson's ratio values.

\section{Conclusions}

A well-defined stress state is found for a conical punch with rounded tip, even for small tip radii, and the implications for indentation testing are discussed. It is found that the radial tensile field, promoting initiation and propulsion of surface cracks, is very insensitive to the sharpness of the cone - indeed it is completely insensitive on the surface. Further, the onset of yielding is predicted at the apex only for very small radius. This indicates that, providing the cone is not too sharp, plastic deformation can be neglected, and the indenter can be considered a good tool for inducing a well-defined, appropriate tensile regime. Finally, concerning the flat indenter case, the geometry is resistant to yielding, unless the corner is particularly sharp $(b / a>0 \cdot 8)$. However there is no particular advantage in using this geometry, as the tensile field is less well-behaved in the region close to the punch edge, so that initiation of cracks and interpretation of results will not be particularly easy.

A complete set of practical diagrams has been given for design of indentation testing experiments has been provided.

\section{Acknowledgements}

Michele Ciavarella is pleased to acknowledge the support from CNR-Consiglio Nazionale delle 
Ricerche (Borsa 203.07.26 del 12.9.96), for his visit to Oxford University, permitting the completion of the present work.

\section{References}

1. Hills, D. A., Dai, D. N. and Warren, P. D., The median crack driven by a point force. Journal of the European Ceramic Society, 1996, 16(11), 1209-1212.

2. Giannakopolous, A. E., Larsson, P. L. and Vestergaard, R., Analysis of Vickers indentation. Int. J. Solids Struct., 1994, 13(19), 2679-2708.

3. Larsson, P. L., Giannakopoulos, A. E., Söderlund, E., Rowecliffe, D. J. and Vestergaard, R., Analysis of Berkovitch indentation. Int. J. Solids Struct., 1996, 32, 221-248.

4. Truman, C. E., Sackfield, A. and Hills, D. A., Contact mechanics of wedge and cone indenters. Int. J. Mech. Sci., 1995, 37(3), 261-275.

5. Dai, D. N., Hills, D. A. and Nowell, D., Formulation and implementation of the Eigenstrain method employing higher-order elements. Int. J. Solids Struct., 1996, 33(3), 331-342.

6. Hills, D. A., Kelly, P. A., Dai, D. N. and Korsunsky, A. M. Solution of Crack Problems: The Distributed Dislocations Technique. Kluwer, Dordrecht, The Netherlands, 1996.

7. Warren, P. D. and Hills, D. A., The influence of elastic mismatch between indenter and substrate on Hertzian fracture. J. of Mat. Sci., 1994, 29(11), 2860-2866.

8. Warren, P. D., Hills, D. A. and Dai, D. N., Mechanics of Hertzian cracking. Tribology international, 1995, 28(6), 357-362.

9. Dai, D. N., Hills, D. A., Warren, P. D. and Nowell, D., The propulsion of surface flaws by elastic indentation testing. Acta Metallurgica et Materalia, 1995, 43(3), 985-991.
10. Ciavarella, M., Indentation by nominally flat or conical indenters with rounded corners. Int. J. Solids Struct., in press.

11. Hübber, 1904.

12. Hills, D. A., Nowell, D. and Sackfield, A., Mechanics of Elastic Contacts. Buttermann-Heinemann, Oxford, 1993.

13. Way, S., Some observation on the theory of contact pressures. J. Appl. Mech. Trans. ASME, 1940, 62, 147-157.

14. Sneddon, I. N. Fourier Transforms. McGraw-Hill, New York, 1951.

15. Timoshenko, S. P. and Goodier, J. N. Theory of Elasticity, 3rd edn. McGraw-Hill, New York, 1970.

16. Shtaerman, I. Ya., Contact Problem of the Theory of Elasticity, Gostekhteoretizdat, Moscow, Leningrad, (Available from the British Library in a English translation by Foreign Technology Div., FTD-MT-24-61-70, 1970.

17. Gladwell, G. M. L., Contact Problems in the Classical Theory of Elasticity. Sitjhoff \& Nordhoff, Alphen aan Olen Rijn.

18. Roesler, F. C., Brittle fracture near equilibrium. Proc. Phys. Soc., 1957, 69, 981-992.

19. Gradshteyn, I. S. and Ryzhik, I. M., Table of Integrals, Series and Products. Academic Press, London, 1980.

20. Love, A. E. H., A Treatise on the Mathematical Theory of Elasticity, 4th ed. Cambridge University Press, Cambridge, 1927.

21. Sachfield and Hills, PA, 1988.

22. Johnson, K. L., Contact Mechanics. Cambridge University Press, Cambridge, 1985.

23. Dundurs, J., Properties of elastic bodies in contact. In The Mechanics of the Contact between Deformable Bodies, ed. A. D. de Pater and J. J. Kalker. Delft University Press, 1975, pp. 54-66.

24. Sneddon, I. N., The relation between load and penetration in the axisymmetric Boussinesq problem for a punch of arbitrary profile. Int. J. Engng. Sci., 1963, 3, 47-57. 